\title{
ALMOST TRIANGULAR MATRICES OVER DEDEKIND DOMAINS
}

\author{
FRANK DEMEYER and HANIYA KAKAKHAIL
}

(Received 25 November 1997 and in revised form 13 March 1998)

\begin{abstract}
Every matrix over a Dedekind domain is equivalent to a direct sum of matrices $A=\left(a_{i, j}\right)$, where $a_{i, j}=0$ whenever $j>i+1$.
\end{abstract}

Keywords and phrases. Matrices, Dedekind domains, equivalence.

1991 Mathematics Subject Classification. 13F05, 15A21.

1. Introduction. Two $m \times n$ matrices $A$ and $B$ over a ring $R$ are called equivalent if $B=P A Q$ for invertible matrices $P$ and $Q$ over $R$. From now on, assume that $R$ denotes a Dedekind domain with quotient field $K$. If $I=\langle a, b\rangle$ is a non principal ideal in $R$, then, in contrast with the situation for Principal Ideal Domains, the $1 \times 2$ matrix $[a, b]$ is not equivalent over $R$ to a matrix whose off diagonal entries are 0 . Using the separated divisor theorem in the form given by Levy in [2], other facts about matrices over Dedekind domains in [2], and elementary properties of ideals in Dedekind domain [1], we show that any $m \times n$ matrix over a Dedekind domain is equivalent to a direct sum of matrices $A=\left(a_{i, j}\right)$ with $a_{i, j}=0$ when $j>i+1$. If the direct summand $A$ has rank $r$, then the number of rows, respectively columns, of $A$ is either $r$ or $r+1$. The corresponding result for similarity of matrices over principal ideal rings is that every $n \times n$ matrix over a principal ideal ring is similar to an upper triangular matrix [3, p. 42].

2. Diagonalization of matrices. If $A$ is an $m \times n$ matrix, then $A$ can be viewed as an $R$-module homomorphism $A: R^{n} \rightarrow R^{m}$ by left multiplication. If $M_{A}$ denotes the submodule of $R^{m}$ generated by the columns of $A$, then $M_{A}$ is the image of $A$ in $R^{m}$ and the isomorphism class of the cokernel $S_{A}=R^{m} / M_{A}$ of $A$ determines the equivalence class of $A$.

SEPARATED DIVISOR THEOREM [2]. There is a chain of integral $R$-ideals $L_{1} \subseteq L_{2} \subseteq$ $\cdots \subseteq L_{r}$ and a fractional $R$-ideal $H$ such that

$$
S_{A}= \begin{cases}\oplus_{i=1}^{r} \frac{R}{L_{i}} \oplus H \oplus R^{m-r-1}, & m<r \\ \oplus_{i=1}^{r} \frac{R}{L_{i}}, & m=r,\end{cases}
$$

where $H=\prod_{i=1}^{r} L_{i}$ if $r=n$ and $H \cong R$ if $r=0$ or $r=m$.

The isomorphism class of $S_{A}$, the ideals $\left\{L_{i}\right\}_{i=1}^{r}$ (as sets), and the isomorphism class of $H$ both determine and are determined by the equivalence class of $A$. 
We also need the following elementary facts about ideals in Dedekind domains.

LEMMA 1 [1, p. 150, 154]. Let $I, J$ be integral ideals in $R$. Then

(1) There is an $\alpha$ in the quotient field $K$ of $R$ such that $\alpha I$ is integral and $\alpha I+J=R$;

(2) There is an $R$-module isomorphism $\gamma: I J \oplus R \rightarrow I \oplus J$, given by $\gamma(u, v)=\left(x_{1} v-u\right.$, $\left.\alpha u-x_{2} v\right)$, where $\alpha$ is as in (1) and $x_{1} \in I, x_{2} \in J$ are chosen with $\alpha x_{1}-x_{2}=1$.

Note. The $R$-linear homomorphism $\gamma$ is given by the matrix $\left(\begin{array}{cc}-1 & x_{1} \\ \alpha & -x_{2}\end{array}\right)$, where $\alpha \in K$.

THEOREM 2.2. Every $m \times n$ matrix $A$ over a Dedekind domain is equivalent to $a$ direct sum of matrices $\left(a_{i j}\right)$ with $a_{i j}=0$ whenever $j>i+1$.

Proof. An $m \times n$ matrix $A$ is called indecomposable if $A$ is not equivalent to a matrix of the form $\left(\begin{array}{cc}B_{1} & 0 \\ 0 & B_{2}\end{array}\right)$ for any matrices $B_{1}, B_{2}$. That is, $A$ is not equivalent to a direct sum of matrices $B_{1}, B_{2}$. If $A=0$, the result is clear. Assume that $A \neq 0$. It is sufficient to verify the result for indecomposable matrices. In this case, if $r$ is the rank of $A$ over the quotient field $K$ of $R$, then [2, Lem. 2.1] asserts that $m=r$ or $r+1$ and $n=r$ or $r+1$. There are then four possible cases to check.

CASE 1. Assume that $m=r$ and $n=r$. Then $S_{A}=\oplus_{i=1}^{r} R / L_{i}$, with $L_{1}, \ldots, L_{r}$ integral $R$-ideals with $L_{1} \subseteq L_{2} \subseteq \cdots \subseteq L_{r}$ and $\prod_{i=1}^{r} L_{i} \cong R$. Thus, $\prod_{i=1}^{r} L_{i}=\langle a\rangle$ is a principal ideal generated by $a \in R$. Let $\phi_{0}: R^{r} \rightarrow \prod_{i=1}^{r} L_{i} \oplus R^{r-1}$ be given by $\phi_{0}\left(r_{1}, \ldots, r_{r}\right)=$ $\left(a r_{1}, r_{2}, \ldots, r_{r}\right)$ and let $\phi_{j}: L_{1} \oplus \cdots \oplus L_{j-1} \oplus \prod_{i=j}^{r} L_{i} \oplus R \oplus R^{r-j-1} \rightarrow L_{1} \oplus \cdots \oplus L_{j} \oplus$ $\prod_{i=j+1}^{r} L_{i} \oplus R^{r-j-1}$ be given by $\phi_{j}=I_{j-1} \oplus \gamma_{j} \oplus I_{r-j-1}$, where $\gamma_{j}: \prod_{i=j}^{r} L_{i} \oplus R \rightarrow L_{j} \oplus$ $\prod_{i=j+1}^{r} L_{i}$ is the map given in Lemma 1 and $I_{j-1}, I_{r-j-1}$ are the identity maps of indicated rank. Let $\phi: R^{r} \rightarrow L_{1} \oplus \cdots \oplus L_{r} \subset R^{r}$ be given by $\phi=\phi_{r-1} \phi_{r-2} \cdots \phi_{1} \phi_{0}$. Then the matrix $[\phi]$ of $\phi$, with respect to the standard bases for $R^{r}$, is: $[\phi]=\left[\phi_{r-1}\right] \cdots[\phi]\left[\phi_{0}\right]$.

While $\left[\phi_{i}\right]$ may have entries which are not in $R,[\phi]$ has all its entries in $R$ since each $L_{j}$ is integral. If we write

$$
\left[\phi_{j}\right]=\left(\begin{array}{cccc}
I_{j} & 0 & 0 & 0 \\
0 & -1 & x_{i}^{j} & 0 \\
0 & \alpha_{j} & -x_{2}^{j} & 0 \\
0 & 0 & 0 & I_{r-j-1}
\end{array}\right)
$$

then a direct calculation shows that

$$
[\phi]=\left(\begin{array}{ccccccccc}
-a & x_{1}^{1} & 0 & 0 & 0 & 0 & 0 & 0 & 0 \\
-a \alpha_{1} & -x_{2}^{1} & x_{1}^{2} & 0 & 0 & 0 & 0 & 0 & 0 \\
-a \alpha_{1} \alpha_{2} & \alpha_{2} x_{2}^{1} & x_{2}^{2} & x_{1}^{3} & 0 & 0 & 0 & 0 & 0 \\
-a \alpha_{1} \alpha_{2} \alpha_{3} & \alpha_{2} \alpha_{3} x_{2}^{2} & x_{2}^{3} & x_{1}^{4} & 0 & 0 & 0 & 0 & 0 \\
\vdots & \vdots & & & & & & & \vdots \\
-a \prod_{i=1}^{r-1} \alpha_{i} & & & \cdots & & & & \alpha_{r-2} x_{2}^{r-2} & x_{2}^{r-1}
\end{array}\right)
$$

Since $[\phi]$ has the same number of rows and columns and the same cokernel as $A$, $[\phi]$ is equivalent to $A$. 
REMARK. Assume that $L_{i}=\left\langle a_{i}\right\rangle$ is principal for each $i, i=1, \ldots, r$ and $a_{i} \in R$. The isomorphism $\gamma_{j}: \prod_{i=j}^{r} L_{i} \oplus R \oplus \rightarrow L_{j} \oplus \prod_{i=j+1}^{r} L_{i}$ can be given as $\gamma_{j}(u, v)=\left(\alpha_{j} u, \beta_{j} v\right)$, where $\alpha_{j}=1 / \prod_{i=j+1} a_{i}$ and $\beta_{j}=\prod_{i=j+1}^{r} a_{i}$. In this case, $[\phi]=\operatorname{diag}\left\{a_{1}, \ldots, a_{r}\right\}$ with $a_{i} \mid a_{i+1}$ for $1 \leq i \leq r$. This is the only case which occurs if $R$ is a PID.

CASE 2. Assume that $m=r$ and $n=r+1$. Then $S_{A}=\oplus_{i=1}^{r} R / L_{i}$ with $L_{i}, 1 \leq i \leq r$ integral ideals and $L_{1} \subseteq L_{2} \subseteq \cdots \subseteq L_{r}$. Let $L_{r+1}$ be integral ideal with $\prod_{i=1}^{r+1} L=\langle a\rangle$ principal, then $\oplus_{i=1}^{r+1} L_{i} \cong R^{n}$ and there is a chain of $R$-homomorphisms

$$
R^{n} \stackrel{\phi}{\longrightarrow} L_{1} \oplus \cdots \oplus L_{r} \oplus L_{r+1} \stackrel{\pi}{\longrightarrow} L_{1} \oplus \cdots \oplus L_{r} \subseteq R^{r},
$$

where $\pi$ is the projection on $L_{1} \oplus \cdots \oplus L_{r}$ along $L_{r+1}$. The matrix of $\pi \circ \phi$ is an $m \times n$ matrix obtained by deleting the last row of $[\phi]$ and, thus, has the same form as in Case 1. Since the cokernel of $\pi \phi$ is the same as $A$ and $[\pi \phi]$ has the same number of rows and columns as $A,[\pi \phi]$ is equivalent to $A$.

CASE 3. Assume that $m=r+1$ and $n=r$. Then $S_{A}=\oplus_{i=1}^{r} R / L_{i} \oplus H$, where $L_{i}, 1 \leq i \leq r$ are integral ideals and $H \cong \prod_{i=1}^{r} L_{i}$. Choose $a \in R$ with $L_{r} H^{-1} a$ integral. Note that $L_{r} H^{-1} a$ is a submodule of $H^{-1} a$. From Case 1 , we construct an $R$-isomorphism $\phi_{r}: R^{r} \rightarrow L_{1} \oplus \cdots \oplus L_{r-1} \oplus L_{r} H^{-1} a \subset R^{r+1}$ whose matrix has the same form as that of $[\phi]$ in Case 1 . By Lemma 1 , there is a chain of isomorphisms $\psi: H^{-1} a \oplus H \rightarrow H^{-1} H a \oplus R \rightarrow R \oplus R$ carrying $L_{r} H^{-1} a$ onto a submodule $N$ of $R \oplus R$. By [1, Cor. 18.24], $\left(H^{-1} a \oplus H\right) / L_{r} H^{-1} a \cong R / L_{r} \oplus H$. Let $\Phi=\left(I_{r-1} \oplus \psi\right) \circ \phi_{r}: R^{n} \rightarrow R^{m}$. The matrix of $\Phi$ is $m \times n$ and the first $r=n$ rows are the same as [ $\phi_{r}$ ]. The last row does not contribute any entries above the main diagonal. So, for each $j>i+1$, the $i, j$ th entry of $[\Phi]$ is 0 . Since the cokernel of $[\Phi]$ is $S_{A}$ and [ $\left.\Phi\right]$ has the same number of rows and columns as $A,[\Phi]$ and $A$ are equivalent.

CASE 4. Let $S_{A}=\oplus_{i=1}^{r} R / L_{i} \oplus H$, where $L_{1}, \ldots, L_{r}$ are integral ideals with $L_{1} \subseteq \cdots \subseteq$ $L_{r}$ and by replacing $H$ (if necessary) by an isomorphic copy, $H$ is an integral ideal. By [1, Thm. 18.20], there is an integral ideal $H_{o}$ with $H_{o} H$ principal and $H_{o}+H=R$. There is an $a \in R$ such that $J=\left(\prod_{i=1}^{r} L_{i} \cdot H_{o}\right)^{-1} a \subseteq H$. As in Case 1 , there is an isomorphism $\phi_{r+1}: R^{r+1} \rightarrow L_{1} \oplus \cdots \oplus L_{r-1} \oplus L_{r} H_{o} \oplus J$. View $L_{i} \leq R$ for $1 \leq i \leq r, L_{r} H_{o} \leq$ $H_{o}$. As in Case 3, there is an isomorphism $\psi: H_{o} \oplus H \rightarrow R \oplus R$ with $\psi\left(L_{r} H_{o}\right)=N \leq R \oplus R$ and $R \oplus R / N \cong R / L_{r} \oplus H$. Let $\Phi=\left(I_{r-1} \oplus \psi\right) \circ \phi_{r+1}$. Then $\Phi: R^{r+1} \rightarrow R^{r+1}$ and all the rows, except possibly the last two of $[\Phi]$, are the same as that of $[\phi]$ in Case 1 . So, for each $j>i+1$, the $i, j$ th entry of $[\Phi]$ is 0 . Since the cokernel of $\Phi$ is $S_{A}$, [ $\left.\Phi\right]$ and $A$ are equivalent.

REMARK. While we could have given explicit formula for the entries in the matrices constructed in Cases 2, 3, and 4 as in Case 1, these entries are not canonically determined by $A$ as a result of the many choices made in their construction. In particular, the choices of $\alpha$ and $x_{1}, x_{2}$ in Lemma 1 are not canonically determined by the ideals $I, J$.

\section{REFERENCES}

[1] C. W. Curtis and I. Reiner, Representation theory of finite groups and associative algebras, Pure and Applied Mathematics, vol. 11, Interscience Publishers, a division of John Wiley \& Sons, New York, London, 1962. MR 26\#2519. Zbl 131.25601. 
[2] L. S. Levy, Almost diagonal matrices over Dedekind domains, Math.-Z. 124 (1972), 89-99. MR 45 3437. Zbl 211.36903.

[3] M. Newman, Integral matrices, Pure and Applied Mathematics, vol. 45, Academic Press, New York, London, 1972. MR 49 5038. Zbl 254.15009.

Demeyer: Department of Mathematics, Colorado State University, Fort Collins CO 80523, USA

E-mail address: demeyer@math.colostate.edu

Kakakhail: Department of Mathematics, Metropolitan State College, Denver CO 80217, USA

E-mail address: mashroor.kakakhe1@uchsc.edu 


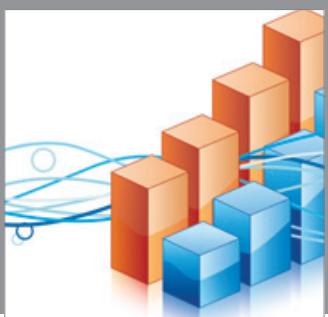

Advances in

Operations Research

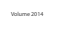

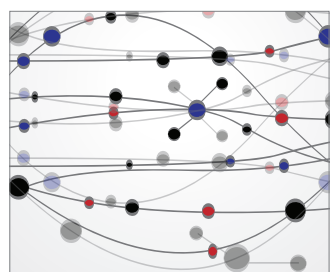

\section{The Scientific} World Journal
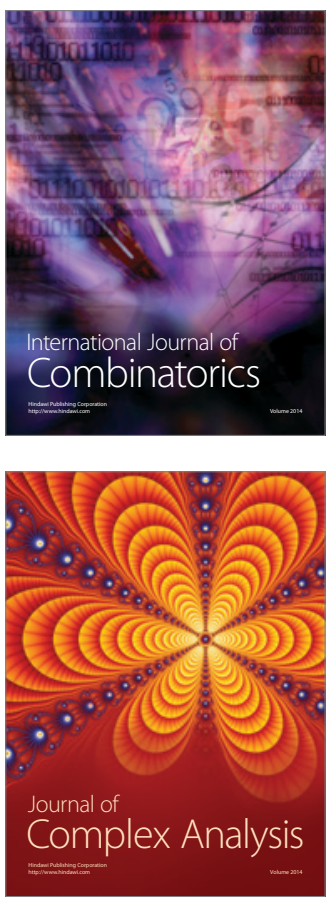

International Journal of

Mathematics and

Mathematical

Sciences
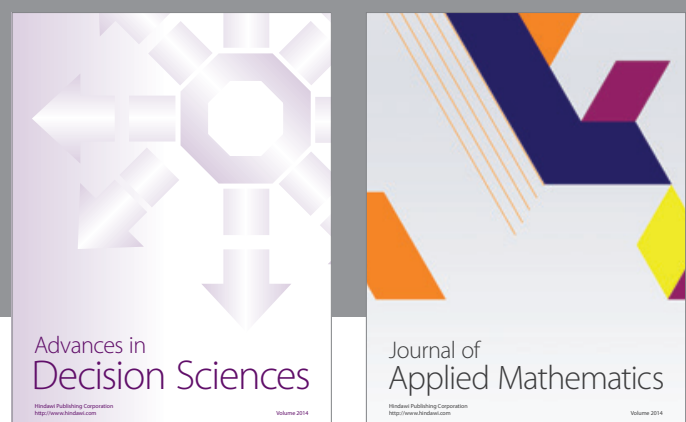

Journal of

Applied Mathematics
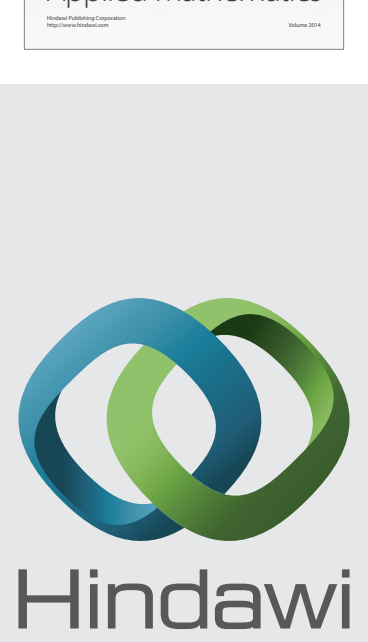

Submit your manuscripts at http://www.hindawi.com
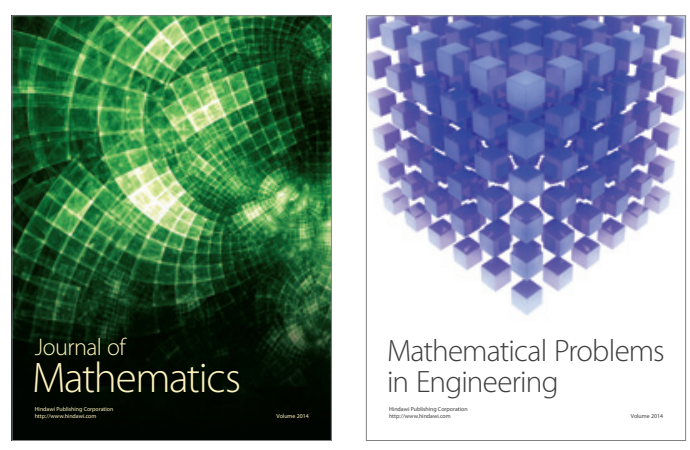

Mathematical Problems in Engineering
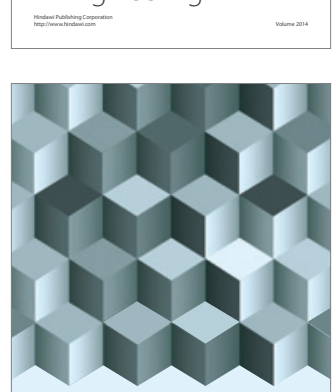

Journal of

Function Spaces
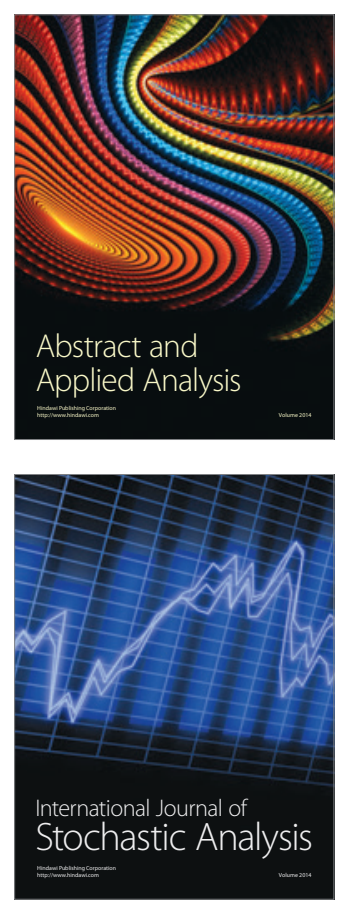

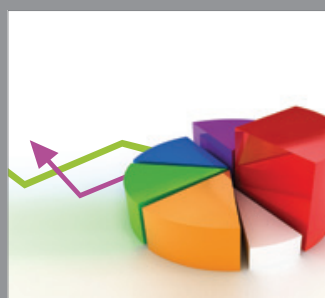

ournal of

Probability and Statistics

Promensencen
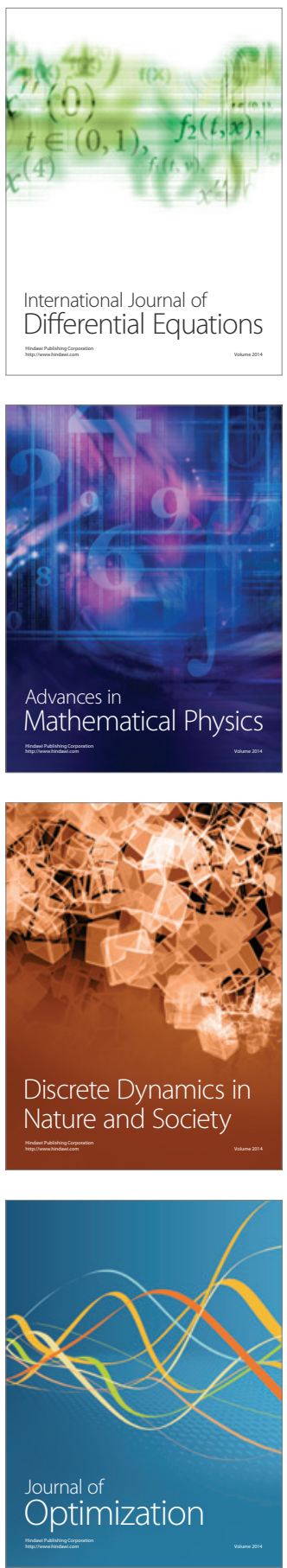